\title{
A converse comparison theorem for BSDEs
}

\author{
Zhihui Zhao, a, Minle Wang ${ }^{2, b}$, Congwei $W^{3, c}$, Yahong Zhu ${ }^{4, d}$ \\ ${ }^{1,2,3,4}$ Department of Mathematics, Xi'an Research Institute of High-tech, Tongxin Road No.2,2102 \\ Team, Baqiao District, Xi'an City, Shaanxi Province, P.R.China, Xi'an 710025 \\ a,b,c,d wucongweihome@sina.com
}

Keywords: Converse comparison theorem, backward stochastic differential equations.

\begin{abstract}
We establish a converse comparison theorem for backward stochastic differential equations(BSDEs). Under some weaker assumptions, we prove that we can compare the generators if we can compare the solutions of two BSDEs (at time $t=0$ ) with the same terminal condition.
\end{abstract}

\section{Introduction}

Backward stochastic differential equations(BSDEs) have been first introduced by Pardoux and Peng [6]. In this paper, they proved that there existed a unique, adapted and square integrable solution to a BSDE of type

$$
y_{t}=\xi+\int_{t}^{T} g\left(s, y_{s}, z_{s}\right) d s-\int_{t}^{T} Z_{s} d W_{s}, \quad 0 \leq t \leq T
$$

Providing that the generator was lipshitz in both variables $y$ and $z$, and that $\xi$ and $(g(s, 0,0))_{s \in[0, T]}$ are square integrable. The comparison theorem, which is an important theory of BSDE, was established by Peng [7] and then generalized by EI Karoui et al.[4]. It allows to compare the solutions of two real-valued BSDEs whenever we can compare the terminal conditions and the generators. In this note, we try to investigate a converse problem: If we can compare the solutions of two BSDEs (at time $t=0$ )with the same terminal condition, for all terminal conditions, can we compare the generators?

Chen [2], Briand et al.[1] and Coquet et al.[3] work on this subject and established some converse comparison theorems. In their papers, the authors all assumed that $g(s, y, 0)=0$. The condition is so strong that many generators do not satisfy it. So Jiang[5] generalized their results under some natural and reasonable assumptions about generator $g$. But it needs that we can compare the solutions of two real-valued BSDEs at any time. It seems to be strong.

In this note, under some weaker assumptions, we prove that we can compare the generators if we can compare the solutions of two BSDEs (at time $t=0$ ) with the same terminal condition.

\section{Preliminaries}

Before giving the main results, let us introduce some notations and assumptions.

Let $(\Omega, F, P)$ be a probability space carrying a standard d-dimensional Brownian motion, $\left(W_{t}\right)_{t \geq 0}$, starting from $W_{0}=0$, and let $\left(F_{t}\right)_{t \geq 0}$ be the $\sigma$-algebra generated by $\left(W_{t}\right)_{t \geq 0}$. We do the usual $P$ augmentation to each $F_{t}$ such that $\left(F_{t}\right)_{t \geq 0}$ is right continuous and complete. If $z$ belong to $R^{d}$, $\|z\|$ denotes its Euclidean norm. We define the following usual spaces of processes:

$$
\begin{aligned}
& S_{2}=\left\{\phi \text { progressivelymeasurable: }\|\phi\|_{S_{2}}^{2}=E\left[\sup _{0 \leq t \leq T}\left|\phi_{t}\right|^{2}\right]<\infty\right\} \\
& H_{2}\left(R^{d}\right)=\left\{\phi \text { progressivelymeasurable: }\|\phi\|_{2}^{2}=E\left[\int_{0}^{T}\left|\phi_{t}\right|^{2} d t\right]<\infty\right\}
\end{aligned}
$$


Let the generator $g$ of a BSDE be a function $g: \Omega \times[0, T] \times R \times R^{d} \rightarrow R$ such that the process $(g(t, y, z))_{t \in[0, T]}$ is progressively measurable for each $(y, z) \in R \times R^{d}$. For the function $g$, we make the following assumptions:

(A1) There exists a constant $K \geq 0$, such that

$$
\left|g\left(t, y_{1}, z_{1}\right)-g\left(t, y_{2}, z_{2}\right)\right| \leq K\left(\left|y_{1}-y_{2}\right|+\left|z_{1}-z_{2}\right|\right) \quad P-\text { a.s. } \forall t, y_{1}, y_{2}, z_{1}, z_{2} ;
$$

(A2) The process $(g(t, 0,0))_{t \in[0, T]} \in H_{2}(R)$;

(A3) $P$-a.s., $\forall(y, z) \in R \times R^{d}, t \rightarrow g(t, y, z)$ is right continuous in $t \in[0, T]$ and left continuous in $T$.

Lemma 2.1 (Pardoux and Peng[6]) Let $g$ satisfy (A1) and (A2), then the BSDE (1) has a unique solution which is a pair of adapted processes $\left(y_{t}(T, g, \xi), z_{t}(T, g, \xi)\right)_{t \in[0, T]}$ in $S_{2} \times H_{2} R^{d}$.

In the following, we often denote $\left(y_{t}(T, g, \xi), z_{t}(T, g, \xi)\right)_{t \in[0, T]}$ by $\left(y_{t}, z_{t}\right)_{t \in[0, T]}$ for convenience.

Lemma 2.2 (Comparison theorem) Let $g, g^{\prime}$ satisfy (A1) and (A2). $y_{T}, y^{\prime}{ }_{T} \in L^{2}\left(\Omega, F_{T}, P\right)$. ( $\left.y, z\right)$ and $\left(y^{\prime}, z^{\prime}\right)$ respectively are the unique adapted solutions of the following two BSDEs:

$$
\begin{gathered}
y_{t}=y_{T}+\int_{t}^{T} g\left(s, y_{s}, z_{s}\right) d s-\int_{t}^{T} z_{s} d W_{s}, \quad 0 \leq t \leq T \\
y_{t}^{\prime}=y_{T}^{\prime}+\int_{t}^{T} g^{\prime}\left(s, y^{\prime}{ }_{s}, z_{s}^{\prime}\right) d s-\int_{t}^{T} z_{s}^{\prime} d W_{s}, \quad 0 \leq t \leq T
\end{gathered}
$$

(1) If $y_{T} \geq y_{T}^{\prime}, g\left(s, y_{s}^{\prime}, z_{s}^{\prime}\right) \geq g^{\prime}\left(s, y_{s}^{\prime}, z_{s}^{\prime}\right)$ a.s., then we have $y_{t} \geq y_{t}^{\prime}$;

(2) Furthermore, $y_{0}=y_{0}^{\prime} \Leftrightarrow y_{T} \geq y_{T}^{\prime}, g\left(s, y_{s}^{\prime}, z_{s}^{\prime}\right) \equiv g^{\prime}\left(s, y_{s}^{\prime}, z_{s}^{\prime}\right)$.

Lemma 2.3 Let $g$ satisfy (A1) and (A2), $0 \leq t \leq s \leq T$ and $\xi \in L^{2}\left(\Omega, F_{T}, P\right)$, then

(1) $y_{t}\left(g, s, y_{s}(g, T, \xi)\right)=y_{t}(g, T, \xi)$;

(2) $y_{t}\left(g, \tau, y_{\tau}(g, T, \xi)\right)=y_{t}(g, T, \xi)$,a.s., if $t \in[0, \tau]$.

\section{Main results}

Theorem 3.1 Let two generators $g_{1}$ and $g_{2}$ satisfy (A1),(A2), and (A3), Then the following two conditions are equivalent:

(i) For any $r \in[0, T], \quad \xi \in L^{2}\left(\Omega, F_{r}, P\right)$ we have $y_{0}\left(g_{1}, r, \xi\right)=y_{0}\left(g_{2}, r, \xi\right)$;

(ii) For any triplet, we have $g_{1}(t, y, z)=g_{2}(t, y, z)$ P-a.s.

Proof. It is obvious that (ii) $\Rightarrow$ (i), so we only need to prove that (i) $\Rightarrow$ (ii).

If we write $y_{0}\left(g_{1}, r, \xi\right)$ as the initial value of the solution of a BSDE, then

$$
y_{0}\left(g_{1}, r, \xi\right)=Y_{0}=\xi+\int_{0}^{r} g_{1}\left(s, Y_{s}, Z_{s}\right) d s-\int_{0}^{r} Z_{s} d W_{s}
$$

where $\left(Y_{s}, Z_{s}\right)$ is the unique adapted solutions of the BSDE.

According to the Lemma 2.3 and the assumption (i), for any $0 \leq r \leq s \leq T$, we get

$$
\begin{aligned}
y_{0}\left(g_{1}, s, \xi\right) & =y_{0}\left(g_{1}, r, y_{r}\left(g_{1}, s, \xi\right)\right) \\
& =y_{0}\left(g_{2}, r, y_{r}\left(g_{1}, s, \xi\right)\right) \\
& =\xi+\int_{r}^{s} g_{1}\left(t, Y_{t}, Z_{t}\right) d t-\int_{r}^{s} Z_{t} d W_{t}+\int_{0}^{r} g_{2}\left(t, Y_{t}, Z_{t}\right) d t-\int_{0}^{r} Z_{t} d W_{t} \\
& =\xi+\int_{0}^{s} g_{1}\left(t, Y_{t}, Z_{t}\right) d t-\int_{0}^{s} Z_{t} d W_{t}+\int_{0}^{r} g_{2}\left(t, Y_{t}, Z_{t}\right) d t-\int_{0}^{r} g_{1}\left(t, Y_{t}, Z_{t}\right) d t \\
& =\xi+\int_{0}^{r}\left(g_{2}\left(t, Y_{t}, Z_{t}\right)-g_{1}\left(t, Y_{t}, Z_{t}\right)\right) d t+\int_{0}^{s} g_{1}\left(t, Y_{t}, Z_{t}\right) d t-\int_{0}^{s} Z_{t} d W_{t} \\
& =y_{0}\left(g_{1}, s, \xi+\int_{0}^{r}\left(g_{2}\left(t, Y_{t}, Z_{t}\right)-g_{1}\left(t, Y_{t}, Z_{t}\right)\right) d t\right)
\end{aligned}
$$

Thus from the Comparison theorem we have 


$$
\left.\xi=\xi+\int_{0}^{r}\left(g_{2}\left(t, Y_{t}, Z_{t}\right)-g_{1}\left(t, Y_{t}, Z_{t}\right)\right) d t\right)
$$

So

$$
\int_{0}^{r}\left(g_{2}\left(t, Y_{t}, Z_{t}\right)-g_{1}\left(t, Y_{t}, Z_{t}\right)\right) d t=0
$$

By the arbitrariness of $r$ and continuity of $g$ in $t$, we have $g_{2}\left(t, Y_{t}, Z_{t}\right)=g_{1}\left(t, Y_{t}, Z_{t}\right)$, $\forall(t, Y, Z) \in[0, T] \times R \times R^{d}$. Thus we complete the proof of Theorem.

Remark 3.1 In the above theorem, we needn't to assume $g(t, 0,0) \equiv 0$. So, our assumptions are more natural and reasonable than that of [5].

For a given stopping time, we now consider the following BSDE

$$
y_{t}=\xi+\int_{t \wedge \tau}^{\tau} g\left(s, t_{s}, z_{s}\right) d s-\int_{t \wedge \tau}^{\tau} z_{s} d W_{s}, \quad \tau \leq T
$$

Remark 3.2 By the result of [8], the Lemma 2.2 still hold true if we consider the above equation(2).

Theorem 3.2 Let two generators $g_{1}$ and $g_{2}$ satisfy (A1), (A2) and (A3). Then the following two conditions are equivalent:

(i) For each stopping time $\tau \leq T, \xi \in L^{2}\left(\Omega, F_{\tau}, P\right)$ we have $y_{0}\left(g_{1}, \tau, \xi\right)=y_{0}\left(g_{2}, \tau, \xi\right)$;

(ii) For any triplet $\forall(t, y, z) \in[0, T] \times R \times R^{d}$, we have $g_{1}(t, y, z)=g_{2}(t, y, z)$ P-a.s.

Proof. It is obvious that (ii) $\Rightarrow$ (i), so we only need to prove that (i) $\Rightarrow$ (ii).

If we write $y_{0}\left(g_{1}, \tau, \xi\right)$ as the initial value of the solution of a BSDE, then

$$
y_{0}\left(g_{1}, \tau, \xi\right)=Y_{0}=\xi+\int_{0}^{\tau} g_{1}\left(s, Y_{s}, Z_{s}\right) d s-\int_{0}^{\tau} Z_{s} d W_{s}
$$

According to the Lemma 2.3 and the assumption (i), for any $0 \leq \tau \leq s \leq T$, we get

$$
\begin{aligned}
y_{0}\left(g_{1}, s, \xi\right) & =y_{0}\left(g_{1}, \tau, y_{\tau}\left(g_{1}, s, \xi\right)\right) \\
& =y_{0}\left(g_{2}, \tau, y_{\tau}\left(g_{1}, s, \xi\right)\right) \\
& =\xi+\int_{\tau}^{s} g_{1}\left(t, Y_{t}, Z_{t}\right) d t-\int_{\tau}^{s} Z_{t} d W_{t}+\int_{0}^{\tau} g_{2}\left(t, Y_{t}, Z_{t}\right) d t-\int_{0}^{\tau} Z_{t} d W_{t} \\
& =\xi+\int_{0}^{s} g_{1}\left(t, Y_{t}, Z_{t}\right) d t-\int_{0}^{s} Z_{t} d W_{t}+\int_{0}^{\tau} g_{2}\left(t, Y_{t}, Z_{t}\right) d t-\int_{0}^{\tau} g_{1}\left(t, Y_{t}, Z_{t}\right) d t \\
& =\xi+\int_{0}^{\tau}\left(g_{2}\left(t, Y_{t}, Z_{t}\right)-g_{1}\left(t, Y_{t}, Z_{t}\right)\right) d t+\int_{0}^{s} g_{1}\left(t, Y_{t}, Z_{t}\right) d t-\int_{0}^{s} Z_{t} d W_{t} \\
& =y_{0}\left(g_{1}, s, \xi+\int_{0}^{\tau}\left(g_{2}\left(t, Y_{t}, Z_{t}\right)-g_{1}\left(t, Y_{t}, Z_{t}\right)\right) d t\right)
\end{aligned}
$$

Thus from the Comparison theorem we have

$$
\left.\xi=\xi+\int_{0}^{\tau}\left(g_{2}\left(t, Y_{t}, Z_{t}\right)-g_{1}\left(t, Y_{t}, Z_{t}\right)\right) d t\right)
$$

So

$$
\int_{0}^{\tau}\left(g_{2}\left(t, Y_{t}, Z_{t}\right)-g_{1}\left(t, Y_{t}, Z_{t}\right)\right) d t=0
$$

By the arbitrariness of $\tau$ and continuity of $g$ in $t$, we have $g_{2}\left(t, Y_{t}, Z_{t}\right)=g_{1}\left(t, Y_{t}, Z_{t}\right)$, $\forall(t, Y, Z) \in[0, T] \times R \times R^{d}$. Thus we complete the proof of Theorem.

Counterexample Let us define generators $g_{1}=y \sin \mathrm{t}$ and $g_{2}=0$. Then $g_{1}, g_{2}$ satisfy assumptions (A1),(A2) and (A3). Let $\left(Y_{t}^{i}, Z_{t}^{i}\right)(i=1,2)$ be the unique adapted solution of equation

$$
Y_{t}^{i}=\xi+\int_{t}^{s} g_{i}\left(r, Y_{r}^{i}, Z_{r}^{i}\right) d r-\int_{t}^{r} Z_{r}^{i} d W_{r}
$$

Applying It's formula to $Y_{t}^{1} e^{\int_{0}^{t} \sin r d r}$, we can get 


$$
d\left(Y_{t}^{1} e^{\int_{0}^{t} \sin r d r}\right)=e^{\int_{0}^{t} \sin r d r} d Y_{t}^{1}+Y_{t}^{1} d e^{\int_{0}^{t} \sin r d r}=e^{\int_{0}^{t} \sin r d r} Z_{t}^{1} d W_{t}
$$

integrate over the interval $[0, s]$, then by taking expectation we conclude that

$$
\begin{gathered}
Y_{0}^{1}=e^{\int_{0}^{s} \sin r d r} E[\xi]=e^{1-\cos s} E[\xi] \\
y_{0}\left(g_{2}, s, \xi\right)=Y_{0}^{2}=E[\xi] \leq e^{1-\cos s} E[\xi]=Y_{0}^{1}=y_{0}\left(g_{1}, s, \xi\right)
\end{gathered}
$$

But the inequality $g_{1} \geq g_{2}$ does not hold.

\section{References}

[1] Briand P., Coquet F., Hu Y., Mmin J., Peng S., A converse comparison theorem for BSDEs and related properties of g-expectation, Electon. Comm. Probab. 2000, 5: 101-117.

[2] Chen Z., A Property of backward stochastic differential equations, C. R. Acad. Sci. Paris, Ser. I 1998, 326(4): 483-488.

[3] Coquet F.,Hu Y., Mmin J.,Peng S., A general converse comparison theorem for backward stochastic differential equations, C. R. Acad. Sci. Paris, Ser. I 2001, 333: 577-581.

[4] EL Karoui N., Peng S., Quenez M.C., Backward stochastic differential equations in finance, Math. Finance 1997, 7(1): 1-71.

[5] Jiang L., Some results on the uniqueness of generators of backward stochastic differential equations, C. R. Acad. Sci. Paris, Ser. I 2004, 338: 575-580.

[6] Pardoux E., Peng S., Adapted solution of a backward stochastic differential equation, Systems Control Lett., 1990, 14: 55-61.

[7] Peng S., A generalized dynamic programming principle and Hamilton-Jacobi-Bellman equation, Stochastics, 1992, 38(2): 119 -134.

[8] Peng S., Monotonic limit theorem of BSDE and nonlinear decomposition theorem of DoobCMeyers type, Probability Theory and Related Fields, 1999, 113(4):473-499. 